\title{
Performance evaluation of selected decision tree algorithms for COVID- 19 diagnosis using routine clinical data
}

\author{
Mostafa Shanbehzadeh ${ }^{1}$, Hadi Kazemi-Arpanahi ${ }^{2,3}$, Raoof Nopour ${ }^{4 *}$ (1) \\ Received: 26 Sep 2020 \\ Published: 1 Mar 2021
}

\section{Abstract}

Background: The novel 2019 Coronavirus disease (COVID-19) poses a great threat to global public health and the economy. The earlier detection of COVID-19 is the key to its treatment and mitigating the transmission of the virus. Given that Machine Learning (ML) could be potentially useful in COVID-19 identification, we compared 7 decision tree (DT) algorithms to select the best clinical diagnostic model.

Methods: A hospital-based retrospective dataset was used to train the selected DT algorithms. The performance of DT models was measured using performance criteria, such as accuracy, sensitivity, specificity, receiver operating characteristic (ROC), and precisionrecall curves (PRC). Finally, the best decision model was obtained based on comparing the mentioned performance criteria.

Results: Based on the Gini Index (GI) scoring model, 13 diagnostic criteria, including the lung lesion existence (GI=0217), fever $(\mathrm{GI}=0.205)$, history of contact with suspected people $(\mathrm{GI}=0.188), \mathrm{O}_{2}$ saturation rate in the blood $(\mathrm{GI}=0.181)$, rhinorrhea $(\mathrm{GI}=0.177)$, dyspnea $(\mathrm{GI}=0.177)$, cough $(\mathrm{GI}=0.159)$, history of taking the immunosuppressive drug $(\mathrm{GI}=0.145)$, history of respiratory failure $(A R D S)(G I=0.141)$, lung lesion situation $(\mathrm{GI}=0.133)$ and appearance $(\mathrm{GI}=0.126)$, diarrhea $(\mathrm{GI}=0.112)$, and nausea and vomiting $(\mathrm{GI}=0.092)$ have been obtained as the most important criteria in diagnosing COVID-19. The results indicated that the J-48, with the accuracy $=0.85, \mathrm{~F}-\mathrm{Score}=0.85, \mathrm{ROC}=0.926$, and $\mathrm{PRC}=0.93$, had the best performance for diagnosing COVID-19.

Conclusion: According to the empirical results, it is promising to implement J-48 in health care settings to increase the accuracy and speed of COVID-19 diagnosis.

Keywords: COVID-19, Novel Coronavirus, Machine Learning, Decision Tree, Data Mining

Conflicts of Interest: None declared

Funding: The Research Deputy of Abadan Faculty of Medical Sciences, Abadan University of Medical Sciences.

*This work has been published under CC BY-NC-SA 1.0 license.

Copyright $\odot$ Iran University of Medical Sciences

Cite this article as: Shanbehzadeh M, Kazemi-Arpanahi H, Nopour R. Performance evaluation of selected decision tree algorithms for COVID-19 diagnosis using routine clinical data. Med J Islam Repub Iran. 2021 (1 Mar);35:29. https://doi.org/10.47176/mjiri.35.29

\section{Introduction}

Since December 2019, a new coronavirus cluster first named SARS-CoV-2 or $2019 \mathrm{n}-\mathrm{CoV}$ has appeared in Wuhan, Republic of China (ROC), and later was known as

Corresponding author: Raoof Nopour, nopour.r@tums.ac.ir

1. Department of Health Information Technology, School of Paramedical, Ilam University of Medical Sciences, Ilam, Iran

2. Department of Health Information Technology, Abadan Faculty of Medical Sciences, Abadan, Iran

3. Student Research Committee, Abadan Faculty of Medical Sciences, Abadan, Iran

4. Department of Health Information Technology and Management, School of Paramedical, Tehran University of Medical Sciences, Tehran, Iran
COVID-19 (1, 2). The World Health Organization (WHO) later on February 11, 2020, declared COVID-19 as a highly contagious viral infectious disease. COVID-

$\uparrow$ What is "already known" in this topic:

COVID-19 is the most dangerous and highly contagious disease with unknown clinical aspects. Currently, in the lack of effective treatment or vaccine, the early diagnosis of COVID19 is the key to its treatment, implement early isolation and quarantine strategies.

$\rightarrow$ What this article adds:

Intelligent and machine learning techniques can be used as an alternative solution in the battle against the COVID-19 pandemic. In this study, we utilized the different decision tree algorithms and compared their performance in diagnosing COVID-19. Based on the results, it can be concluded that the decision tree algorithms can be used as a potential diagnostic model for earlier detection of COVID-19. 
19 initially affects parts of the upper respiratory tract and causes very mild to severe manifestations, such as rhinorrhea, chill, headache, sore throat, dizziness, cough, dyspnea, fever, tiredness, myalgia, gastrointestinal complications, and pneumonia (3-5).

Deadly complications, high infectivity, long latency period, difficultie s of identification and testing, many unknown characteristics, ambiguous transmission modes, differential diagnosis with other upper respiratory conditions, and absence of specific immunization and treatment have increased the challenges of controlling and managing this public health emergency (6). Also, due to the daily exponential growth rates and high rate of mortality, particularly in susceptible individuals, early and accurate identification of COVID-19 is of high importance (7).

Hence, it necessary to seek early detection and isolation of positive cases as rapidly and accurately as possible for containing the transmission of the virus, especially for asymptomatic carriers in an early stage. Using computational solutions and applications for analyzing big datasets is essential for optimal prevention, screening, treatment, and tracing of COVID-19 (8-10). This led to reducing uncertainty and ambiguity by offering diagnosis models for risk analysis, prediction, and care plans $(10,11)$. Also, it can greatly contribute to identifying high-risk groups, early detection of disease, and adoption of effective treatment plans $(12,13)$.

To this end, innovations in information technologies such as artificial intelligence (AI) and big data mining can be applied to improve the quality of health care services by providing users with a wide variety of digital services such as decision support systems $(14,15)$. These technologies have the potential to improve the monitor human mobility patterns, social distancing, epidemic tracking, contact tracing, and real time and continuous health monitoring $(16,17)$.

Applied to the current COVID-19 crisis, the Clinical Decision Support System (CDSS) based on Machine Learning (ML) can be leveraged to increase the quality of care. In this regard, accurate and valid diagnostic support tools to provide patient risk classification and support reliable clinical decision making with the hope of improving patient outcomes and quality of care can help reduce misdiagnosis and poor prognosis (18-20).

Many pieces of evidence have proven that the ML techniques can meet the requirements of classification data modeling, and it has become the standard approach for developing an accurate diagnostic model that can effectively predict prognosis and monitoring of COVID-19 patients $(21,22)$. In this study, we provided a prediction model for the detection of COVID-19 by comparing the performance of the various decision tree (DT) techniques, including decision stump, Hoeffding tree, J-48, the logistic model tree (LMT), random tree, random forest (RF), and REP-tree algorithms, and selecting the best decision model.

\section{Methods}

A cross-sectional retrospective study was conducted in 2020, consisting of 4 sequential steps as follows:

\section{Data Collection}

The COVID-19 retrospective data repository used in the study was obtained from Taleghani hospital, affiliated to Abadan University of Medical Sciences, the focal point center for COVID-19 care and treatment in the southwest of Khuzestan province, south of Iran. A total of 430 case records was included in the present study. The experimentation is ethic compliant and has been approved a certificate of ethics (code: IR.ABADANUMS.REC.1399.128) by the ethics committee board of the Abadan University of Medical Sciences. After a quantitative analysis of medical records, 30 incomplete case records that had a lot of missing data (more than $70 \%$ missing) were excluded from the analysis. Finally, 400 records were remained (250 [62.5\%] as confirmed positive cases, and 150 [37.5\%] as healthy people).

\section{Feature selection}

To make an efficient decision tree model, the number of features used in this research had to be reduced. To reduce the dimensions of the dataset and improve the efficiency of DT algorithms, first, the possible diagnostic criteria for COVID-19 were scored using the Gini Index (GI) measured in RapidMiner Studio Professional software V 7.1.001. Also, to perform effective statistical analysis to make a high-performance diagnostic model according to Formula 1, the number of samples should be 30 times larger than the diagnostic criteria. Therefore, with consideration of the number of the samples $(n=400)$, the number of diagnostic criteria $(n=40)$ had been reduced to 13 . (The Y and X are the numbers of samples and features, respectively).

\section{Formula 1: $\mathrm{Y}=\mathrm{X} * 30: 400=\mathrm{X} * 30 \rightarrow \mathrm{X}=13.33$.}

\section{Model Construction and Assessment}

The DT classification algorithms of Weka application software version 3.9 was used for data mining. These $\mathrm{ML}$ algorithms included decision stump, Hoeffding tree, J-48, LMT, random forest, random tree, and REP-tree. Of the data, $70 \%$ and $30 \%$ were used for model training and testing, respectively. Finally, the most appropriate decision tree algorithm was used to create a diagnostic model of COVID-19 based on the confusion matrix. For the prediction process, each sample can be classified into 2 classes: COVID-19 and non-COVID-19. This confusion matrix consists of 4 elements: true positive (TP), true negative (TN), false positive (FP), and false negative (FN).

The true positive (TP) and true negative (TN) represent the number of samples that belonged to sick and healthy individuals, which are correctly classified by the model, respectively. The false positive (FP) represents the number of healthy individuals that are falsely classified as the patient by the model. The false negative (FN) represents those patients who have incorrectly been classified as healthy people in the model. To evaluate the predictive models, we applied some evaluation measures, including precision (the probability that a positive prediction is correct), sensitivity (the proportion of positive cases classified correctly), accuracy (the fraction of cases classified 


\begin{tabular}{lc} 
Table 1. Calculation of the Performance Metric \\
\hline Performance Criteria & Calculation \\
\hline Accuracy & $\frac{\boldsymbol{T P}+\boldsymbol{T N}}{\boldsymbol{T P}+\boldsymbol{F P}+\boldsymbol{T N}+\boldsymbol{F N}}$ \\
Precision & $\frac{\boldsymbol{T P}}{\boldsymbol{T P}+\boldsymbol{F P}}$ \\
Sensitivity/ Recall & $\frac{\boldsymbol{T P}}{\boldsymbol{T P}+\boldsymbol{F N}}$ \\
Specificity & $\frac{\boldsymbol{T N}}{\boldsymbol{F P}+\boldsymbol{T N}}$ \\
F-score & $2 *\left(\frac{\text { Precision } * \text { Sensitivity }}{\text { Precision }+ \text { Sensitivity }}\right)$ \\
\hline
\end{tabular}

correctly), F-measure (the probability that a positive prediction is correct), receiver operator characteristics (ROC), and precision-recall curves (PRC) to measure the model's performance. Finally, all of these evaluation criteria were compared in terms of the performance to get the best model for diagnosing COVID-19 (Table 1).

\section{Results}

The results of the research data samples classified by DT algorithms by confusion matrix before feature selection have been presented in Table 2.

Based on Table 2, precision, sensitivity, accuracy, Fmeasure, ROC, and PRC are depicted in Table 3.

Based on the information provided by Table 3, and considering the different evaluation criteria of the DT algorithms, in general, the $\mathrm{J}-48$ algorithms with the precision $=$ 0.625 , sensitivity $=0.714$, accuracy $=0.758$, F-score $=$ $0.666, \mathrm{ROC}=0.802$, and $\mathrm{PRC}=0.758$, compared with the other DT algorithms, had better performance before the feature selection process. Also, the Hoeffding tree had the highest precision comparing the other DT algorithms.

After weighing the variables according to the GI measure, the most important diagnostic criteria were obtained and presented in Table 4.

The results of classifying the samples by DT mining algorithms are presented in Table 3. It has been obtained by the most important features in the previous step (13 features), and the characteristics of algorithms were used in the Weka 3.9 software to achieve the maximum efficiency. All data mining algorithms along with their perfor- mance criteria, such as TP, FP, FN, and TN, have been demonstrated in Table 5.

The result of the precision, sensitivity, and accuracy of these algorithms are presented in Table 6.

The results of comparing selected DT algorithms by 3 criteria in Table 6 showed that the decision stump had the highest precision (1), the LMT tree had the best sensitivity value (0.848), and finally, the J-48 algorithm had the highest accuracy rate $(0.85)$ and best classification performance relatively. Figure 1 shows the F-Score and Figure 2 displays the ROC and PRC of all algorithms based on the confusion matrix measures.

As seen in Figure 1, the J-48 algorithm with the F-Score of 0.85 had a better performance than other DT algorithms. The ROC and PRC values of these algorithms have been presented in Figure 2.

According to Figure 2, the J-48 decision tree algorithm with the ROC and PRC of 0.93 and 0.926 was considered the best algorithm in terms of performance. Based on the results of comparing the selected DT algorithms performances, the J-48 algorithm (before and after feature selection) has been considered as the best decision support model for diagnosing COVID-19. In Figure 3, the J-48 with a confidence factor of 0.15 has been implemented in Weka software.

This J-48 algorithm had 9 classification spots (leaf nodes) and was created with a size of 17 (maximum efficiency). The results of $\mathrm{J}-48$ classification performance showed that the diagnostic criterion of lung lesion (root node), which had acquired the highest value of GI score,

\begin{tabular}{lllll} 
Table 2. Results of the Sample Classification by DT Algorithms & & FP & FN \\
\hline No. & Algorithms & TP & 16 & 37 \\
2 & Decision stump & 39 & 5 & TN \\
3 & Hoeffding Tree & 35 & 18 & 40 \\
4 & J-48 & 30 & 22 & 12 \\
5 & LMT & 21 & 25 & 12 \\
6 & Random forest & 25 & 25 & 18 \\
7 & Random tree & 28 & 51 & 15 \\
\end{tabular}

\begin{tabular}{|c|c|c|c|c|c|c|c|}
\hline No. & Algorithms & Precision & Sensitivity & Accuracy & F-Score & ROC & PRC \\
\hline 1 & Decision stump & 0.709 & 0.513 & 0.558 & 0.588 & 0.694 & 0.631 \\
\hline 2 & Hoeffding tree & 0.875 & 0.466 & 0.625 & 0.608 & 0.731 & 0.686 \\
\hline 3 & $\mathrm{~J}-48$ & 0.625 & 0.714 & 0.758 & 0.666 & 0.802 & 0.758 \\
\hline 4 & LMT & 0.488 & 0.636 & 0.716 & 0.552 & 0.653 & 0.606 \\
\hline 5 & Random forest & 0.5 & 0.581 & 0.641 & 0.533 & 0.662 & 0.587 \\
\hline 6 & Random tree & 0.528 & 0.651 & 0.666 & 0.583 & 0.696 & 0.654 \\
\hline 7 & REP-Tree & 0.462 & 0.581 & 0.608 & 0.514 & 0.657 & 0.596 \\
\hline
\end{tabular}




\begin{tabular}{|c|c|c|c|c|c|c|}
\hline No. & Variable Name & Variable Type & Variable Features & & \multicolumn{2}{|c|}{ Gini Index Score } \\
\hline 1 & Lung lesion existence & Binominal & $\begin{array}{c}\text { No } \\
\text { Yes }\end{array}$ & & & \\
\hline 2 & Fever & Binominal & $\begin{array}{l}\text { No } \\
\text { Yes }\end{array}$ & & & \\
\hline 3 & History of contact with suspected people & Binominal & $\begin{array}{l}\text { No } \\
\text { Yes }\end{array}$ & & & \\
\hline 4 & $\mathrm{O}_{2}$ saturation in blood & Poly nominal & $\begin{array}{l}>95 \% \\
85 \%-95 \% \\
<85 \%\end{array}$ & & & \\
\hline 5 & Rhinorrhea & Binominal & $\begin{array}{l}\text { No } \\
\text { Yes }\end{array}$ & & & \\
\hline 6 & Dyspnea & Binominal & $\begin{array}{l}\text { No } \\
\text { Yes }\end{array}$ & & & \\
\hline 7 & Cough & Binominal & $\begin{array}{l}\text { No } \\
\text { Yes }\end{array}$ & & & \\
\hline 8 & History of taking the immunosuppressive drug & Binominal & $\begin{array}{l}\text { No } \\
\text { Yes }\end{array}$ & & & \\
\hline 9 & History of respiratory failure (ARDS) & Binominal & $\begin{array}{l}\text { No } \\
\text { Yes }\end{array}$ & & & \\
\hline 10 & Lung lesion situation & Poly nominal & $\begin{array}{l}\text { Central } \\
\text { Spreading } \\
\text { Compound }\end{array}$ & & & \\
\hline 11 & Lung lesion appearance & Poly nominal & $\begin{array}{l}\text { GGO } \\
\text { Consolidation } \\
\text { Compound }\end{array}$ & & & \\
\hline 12 & Diarrhea & Binominal & $\begin{array}{l}\text { No } \\
\text { Yes }\end{array}$ & & & \\
\hline 13 & Nausea and vomiting & Binominal & $\begin{array}{l}\text { No } \\
\text { Yes }\end{array}$ & & & \\
\hline No & Important $\mathrm{Cl}$ & stics to Build the Model & $\mathrm{TP}$ & $\mathrm{FP}$ & $\mathrm{FN}$ & $\mathrm{TN}$ \\
\hline 1 & Decision stump & $\begin{array}{l}\text { size }=100 \\
\text { l places }=2 \\
\text { aken }=0 \mathrm{~s}\end{array}$ & 44 & 0 & 32 & $\overline{44}$ \\
\hline 2 & $\begin{array}{r}\text { Hoeff } \\
\text { Numl } \\
\text { Spl }\end{array}$ & $\begin{array}{l}\text { Threshold }=0.05 \\
\text { ecimal places }=2 \\
\text { lence }=1.0 \mathrm{E}-7 \\
\text { ken }=0.01 \mathrm{~s}\end{array}$ & 44 & 0 & 31 & 45 \\
\hline 3 & $\begin{array}{r}\mathrm{Co} \\
\text { Numl } \\
\text { The min }\end{array}$ & $\begin{array}{l}\text { factor }=0.15 \\
\text { ecimal places }=3 \\
\text { of folds }=3 \\
\text { umber of object }=2 \\
\text { ken }=0.01 \mathrm{~s}\end{array}$ & 34 & 10 & 8 & 68 \\
\hline 4 & $\begin{array}{l}\text { The minin } \\
\text { Numb }\end{array}$ & $\begin{array}{l}\text { nber of instances }=15 \\
\text { ecimal places }=3 \\
\text { xen }=0.92 \mathrm{~s}\end{array}$ & 28 & 16 & 5 & 71 \\
\hline 5 & $\begin{array}{r}\text { The } \mathrm{n} \\
\mathrm{T} \\
\text { Numb }\end{array}$ & $\begin{array}{l}\text { f iteration }=100 \\
\text { number }=1 \\
\text { ecimal places }=3 \\
\text { ken }=0.02 \mathrm{~s}\end{array}$ & 33 & 11 & 10 & 66 \\
\hline 6 & The minim & $\begin{array}{l}\text { ance portfolio }=0.001 \\
\text { ecimal places }=2 \\
\text { ken }=0.01 \mathrm{~s}\end{array}$ & 32 & 12 & 11 & 65 \\
\hline 7 & Numb & $\begin{array}{l}\operatorname{ling}=\text { false } \\
\text { cimal places }=2 \\
\text { of folds }=3 \\
\text { ken }=0.03 \mathrm{~s}\end{array}$ & 34 & 10 & 9 & 67 \\
\hline
\end{tabular}

could be considered as the most important criterion in the decision tree configuration so that the 148 (37\%) positive samples have been classified in this node place. We interpreted the 2 rules extracted from this algorithm as bellow:

Rule 1: IF (Lung lesion $=$ No \& \& Fever $==$ No) THEN
COVID-19=0;

Rule 2: IF (Lung lesion $=$ No $\& \&$ Fever $==$ Yes $\& \&$ History of contact $==$ No $\& \&$ Cough $==$ No) THEN COVID-19=0. 


\begin{tabular}{|c|c|c|c|c|}
\hline No. & Algorithms & Precision & Sensitivity & Accuracy \\
\hline 1 & Decision stump & 1 & 0.578 & 0.733 \\
\hline 2 & Hoeffding tree & 0.822 & 0.586 & 0.741 \\
\hline 3 & $\mathrm{~J}-48$ & 0.772 & 0.809 & 0.850 \\
\hline 4 & LMT & 0.633 & 0.848 & 0.825 \\
\hline 5 & Random forest & 0.75 & 0.767 & 0.825 \\
\hline 6 & Random tree & 0.727 & 0.744 & 0.808 \\
\hline 7 & REP-Tree & 0.772 & 0.79 & 0.841 \\
\hline
\end{tabular}

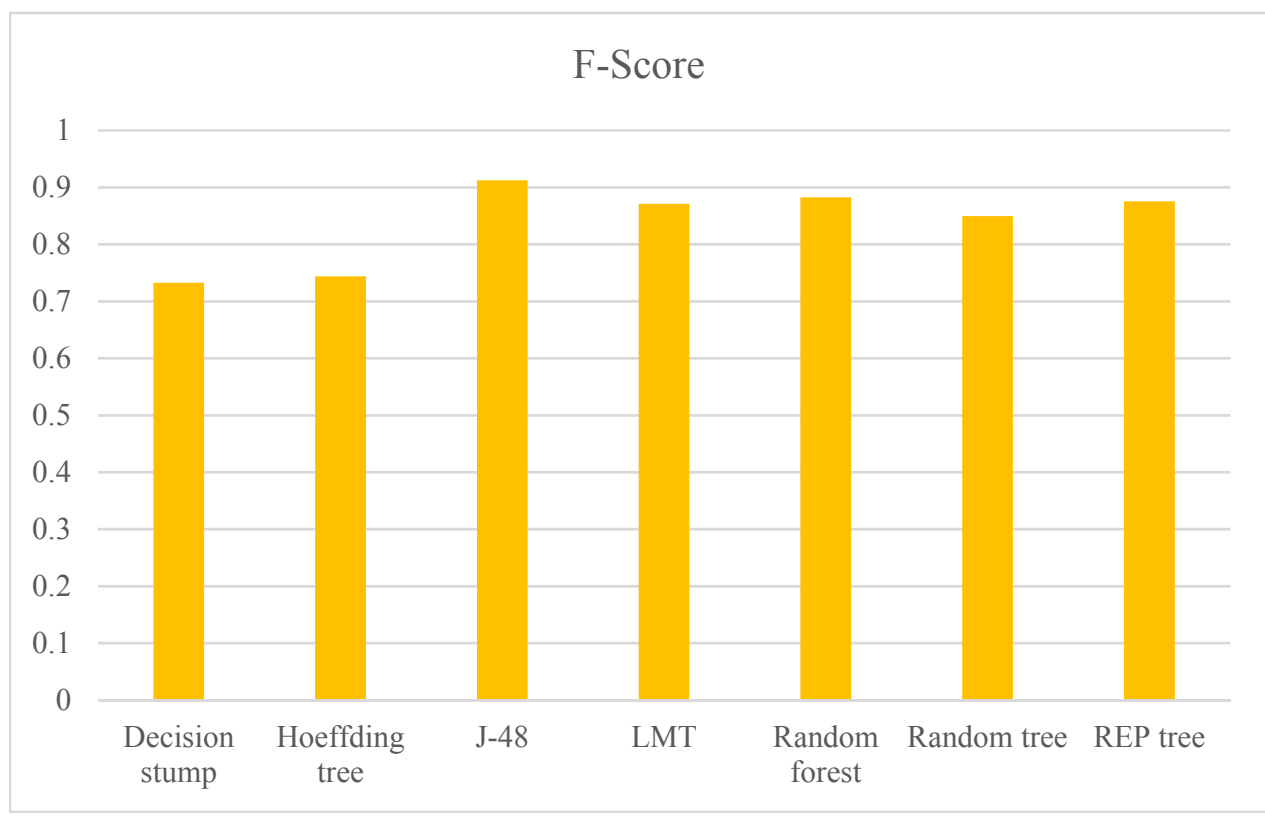

Fig. 1. The Resulted F-Score of the Different Decision Tree Algorithms

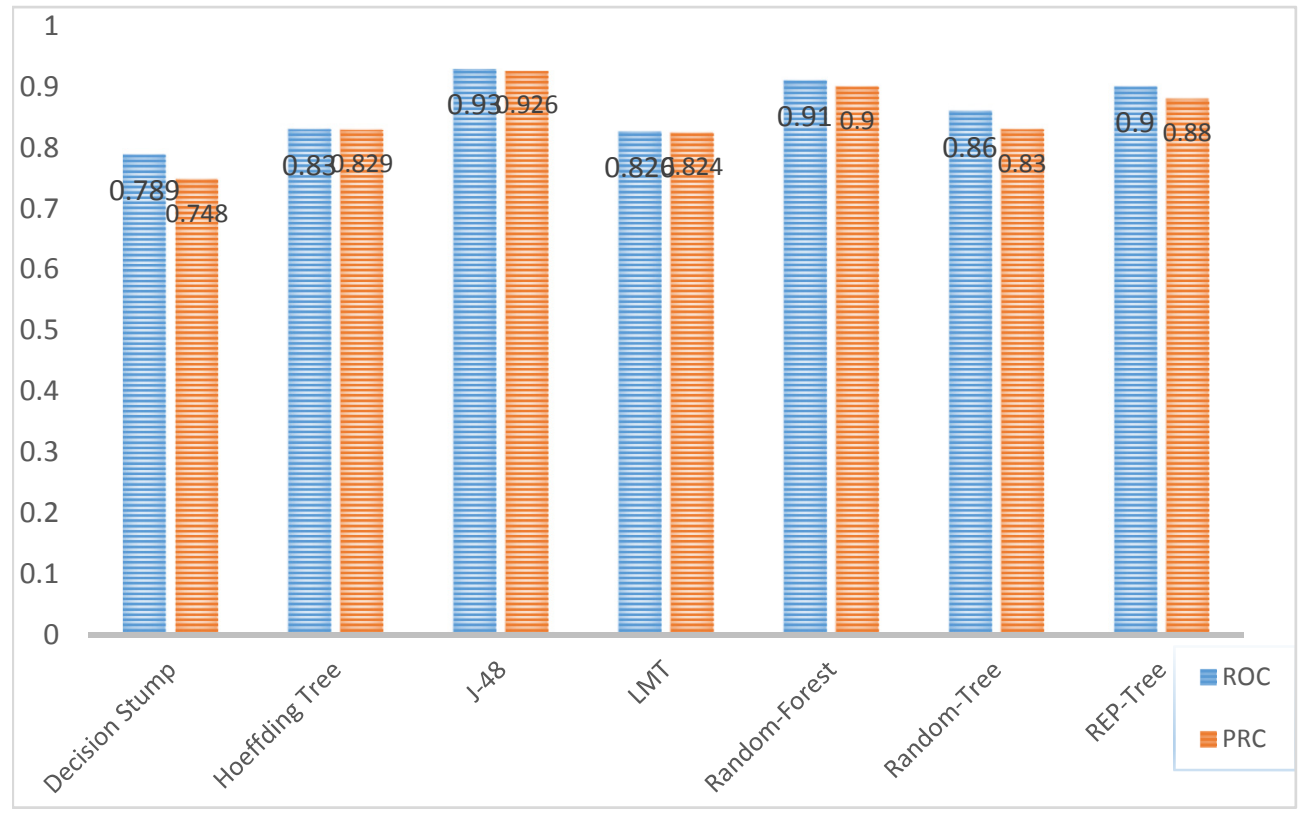

Fig. 2. The ROC and PRC Values of Selected Algorithms

In the first rule, out of 100 samples, $15 \%$ were incorrectly classified (with a positive result). Therefore, with the probability of $85 \%$, this model could detect the negative cases correctly. If a person had not lung lesions and fever, the decision model considered the non-COVID-19 cases with a probability of $85 \%$. In the second rule, out of the 32 samples, sex samples were incorrectly classified ( $81 \%$ of the cases were correctly classified with negative diagnosis), so if a person has no lung lesion but has fever and cough, without the contact history, this decision mod- 


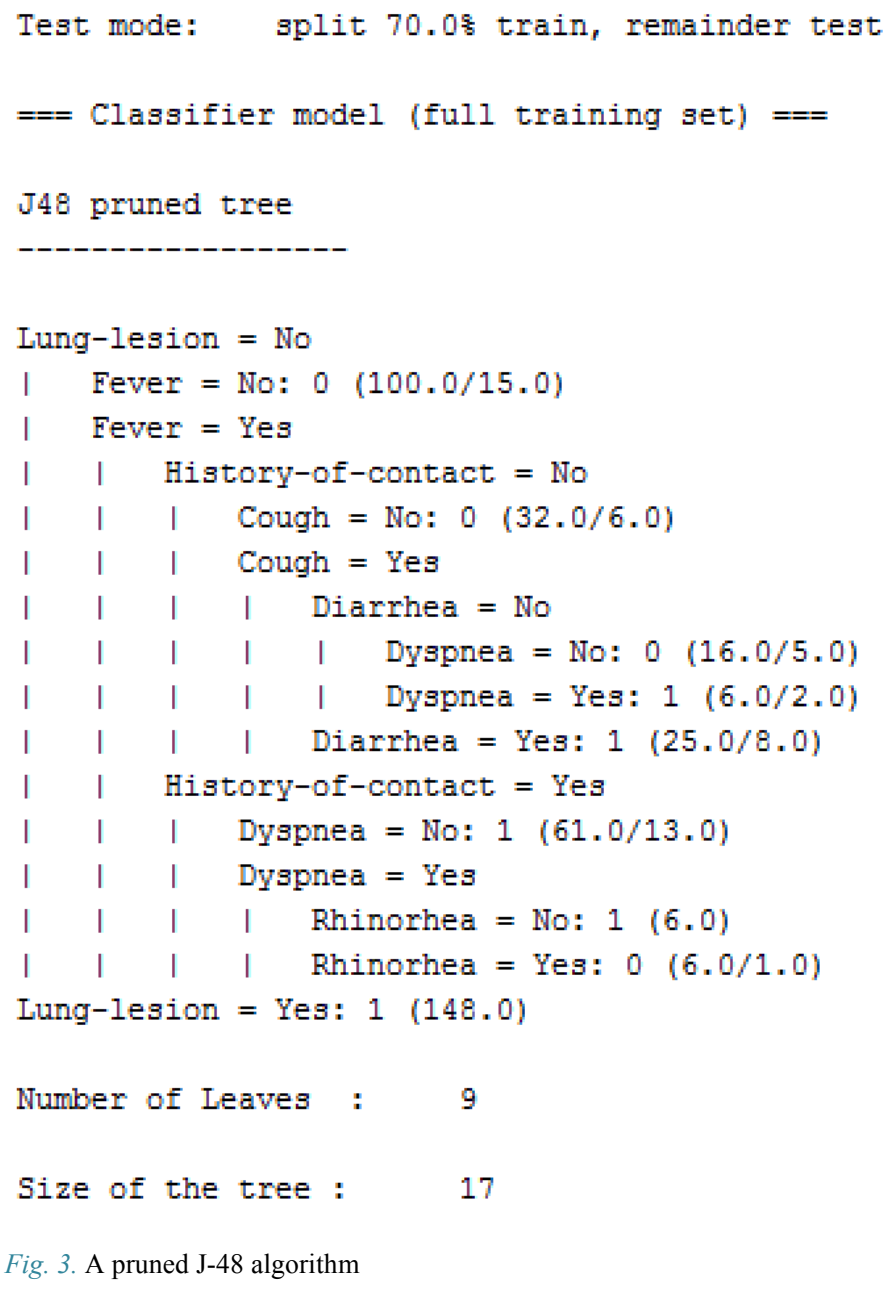

Fig. 3. A pruned J-48 algorithm

el considers this person in the non-COVID-19 group with a probability of the $81 \%$.

\section{Discussion}

COVID-19 is a highly contagious disease and until now continues to spread violently around the world (23-25). The massive outbreak of the COVID-19 and the continuous increased number of infected cases and mortalities have occurred while there is no effective treatment or vaccine (26). Thus, the health care systems around the world are imposing great challenges with severe shortages of required resources and exhaustion of the health care providers (27).

To respond to this ongoing pandemic, many health care authorities across the world have suggested various innovative analytical techniques, such as computational and statistical technologies for real time and accurate disease prediction, screening, diagnosis, and epidemic tracing (26, 28). A timely and accurate diagnosis can provide a better plan for health policymakers and clinicians to mitigate disease outbreaks and improved patient survival probability. To this end, developing prediction models for COVID-19 diagnosis is very crucial in determining their new cases at an early stage $(20,29)$. Health care industries all over the world have employed various technological innovations, such as ML-based artificial intelligence (AI) solution, to fight against COVID-19 (29). Predictive models based on ML for mining datasets can greatly contribute to recognizing high-risk groups, early detection of disease, and adoption of effective treatment plans $(18,30)$. This led to reducing uncertainty and ambiguity by offering evidence-based medicine for risk analysis, prediction, and treatment (11).

The use of DT-based ML algorithms (Learning trees) is proven to be useful for optimal infectious disease prediction and diagnosis $(30,31)$. This led to reducing uncertainty and ambiguity by offering evidence-based medicine for risk analysis, prediction, and care plans (32). Furthermore, DSS-based DT would assist health care providers to make better decisions concerning COVID-19 (diagnosis, classification, etc.) (33). Hence, the purpose of this study was to compare the selected machine learning methods to design a diagnostic expert model based on the DT algorithms for early and accurate disease diagnosis. The main goal of this study was to compare the prediction performance of some decision tree algorithms such as $\mathrm{J}-48$, Bayesian Net, random forest, and multilayer perceptron. The predictive models of our study can discriminate COVID-19 versus non-COVID-19 cases.

Several types of research have been focused on applying and evaluating DT techniques in COVID-19 early progno- 
sis, risk assessment, and trend estimation. Vinod et al (2020) used a DT classifier for recognizing infected cases. The experimental results indicate that this algorithm, with an accuracy of $93 \%$ and a precision of $88 \%$, has the acceptable capability for COVID-19 diagnosis and screening (33). Yoo et al (2020) compared the performance of 3 binary deep learning-based decision-tree classifiers (AXIR1, AXIR2, and AXIR3) for detecting COVID-19. The accuracy of the first, second, and third classifiers was $98 \%, 80 \%$, and $95 \%$, respectively (31). Singer (2020) assessed the performance of 2 ordinal and nonordinal decision-tree-based approaches to estimate the daily local growth rate of the COVID-19 epidemic. They showed that ordinal classifiers yielded better classification results than their nonordinal counterparts (30). Elshazli (2020) showed that the decision tree model (sensitivity $=100 \%$; specificity $=81 \%$ ) has a very good performance in prognosing COVID-19 and identifying high-risk patients (34). Yeşilkanat et al (2020) in their study evaluated the performance of the selected DT algorithms in estimating the near future COVID-19 cases. Finally, the RF algorithm gained the best performance $(\mathrm{R} 2=0.959$ and $\mathrm{RMSE}=259.38)(35)$. The experimental results in the present study indicate that our suggested model (J-48 algorithm) can distinguish between patients and healthy cases at an accuracy of $0.85 \%$, F-Score of $0.85 \%$, ROC of $0.926 \%$, and PRC of $0.93 \%$, producing very successful results in COVID-19 diagnosis.

\section{Limitation}

This study has several challenges and limitations that need to be addressed. A crucial initial challenge in this work was removing noises and incomplete records as much as possible from the dataset. The major limitation was the size of the dataset ( 400 case record). The sample size of the study was not large enough; therefore, it may affect the performance metrics of the diagnostic model. Finally, the dataset lacks some important diagnostic criteria such as laboratory indicators that have an impact on COVID-19 diagnosis. In future research, the scope of application of the model should be expanded by incorporating more comprehensive and qualitative training data. Also, further studies need to be performed with more optimized clinical parameters, bigger and multicenter databases about exploiting more machine learning algorithms.

\section{Conclusion}

In this paper, the efficiency and performance of some DT classifier algorithms were evaluated and compared to the prediction of COVID-19 by using validated clinical features. The results showed that the J-48 was more efficient than the other 6 DT algorithms. The comparison results of diagnostic models' performance in this study were satisfactory to some extent, and we believed that this process will be improved if we increase the number of input variables, and test more machine learning algorithms at the larger, multicenter, and more qualitative dataset.

\section{Acknowledgement}

The research deputy of Abadan University of Medical
Sciences (ethical code: IR. ABADANUMS, REC. 1399128) approved this study. All participants signed an informed consent form before participating in the study. We thank the research deputy of Abadan Faculty of Medical Sciences for financially supporting this project.

\section{Conflict of Interests}

The authors declare that they have no competing interests.

\section{References}

1. Rao ASS, Vazquez JA. Identification of COVID-19 can be quicker through an artificial intelligence framework using a mobile phonebased survey when cities and towns are under quarantine. Infect Control Hosp Epidemiol. 2020;41(7):826-30.

2. Chan JFW, Yuan S, Kok KH, To KKW, Chu H, Yang J, et al. A familial cluster of pneumonia associated with the 2019 novel coronavirus indicating person-to-person transmission: a study of a family cluster. Lancet. 2020;395(10223):514-23.

3. Peeri NC, Shrestha N, Rahman MS, Zaki R, Tan Z, Bibi S, et al. The SARS, MERS, and novel coronavirus (COVID-19) epidemics, the newest and biggest global health threats: what lessons have we learned? Int J Epidemiol. 2020.

4. Mackenzie JS, Smith DW. COVID-19-A Novel Zoonotic Disease: A Review of the Disease, the Virus, and Public Health Measures. Asia Pac J Public Health. 2020;32(4):145-53.

5. Yoo HS, Yoo D. COVID-19 and veterinarians for one health, zoonotic- and reverse-zoonotic transmissions. Vet Sci. 2020;21(3):e51.

6. Saba AI, Elsheikh AH. Forecasting the prevalence of COVID-19 outbreak in Egypt using nonlinear autoregressive artificial neural networks. Process Saf Environ Prot. 2020.

7. Wong MC, Teoh JY, Huang J, Wong SH. Strengthening early testing and surveillance of COVID-19 to enhance the identification of asymptomatic patients. J Infect. 2020;81(2):e112-e3.

8. Agbehadji IE, Awuzie BO, Ngowi AB, Millham RC. Review of Big Data Analytics, Artificial Intelligence, and Nature-Inspired Computing Models towards Accurate Detection of COVID-19 Pandemic Cases and Contact Tracing. Int J Environ Res Public Health. 2020;17(15).

9. Haleem A, Javaid M, Khan IH, Vaishya R. Significant Applications of Big Data in COVID-19 Pandemic. Indian J Orthop. 2020;54(4):526-8.

10. Briz-Redon A, Serrano-Aroca A. The effect of climate on the spread of the COVID-19 pandemic: A review of findings, and statistical and modelling techniques. Prog Phys Geog: Earth and Envr. 2020; 44.5: 591-604.

11. Rehm GB, Woo SH, Chen XL, Kuhn BT, Cortes-Puch I, Anderson NR, et al. Leveraging IoTs and Machine Learning for Patient Diagnosis and Ventilation Management in the Intensive Care Unit. IEEE Pervas Comput. 2020.

12. Shipe ME, Deppen SA, Farjah F, Grogan EL. Developing prediction models for clinical use using logistic regression: an overview. J Thorac Dis. 2019;11(Suppl 4): S574.

13. Sarkodie SA, Owusu PA. Investigating the cases of novel coronavirus disease (COVID-19) in China using dynamic statistical techniques. Heliyon. 2020;6(4).

14. Bansal A, Padappayil RP, Garg C, Singal A, Gupta M, Klein A. Utility of Artificial Intelligence Amidst the COVID 19 Pandemic: A Review. J Med Syst. 2020;44(9).

15. Vinod DN, Prabaharan SRS. Data science and the role of Artificial Intelligence in achieving the fast diagnosis of Covid-19. Chaos Solitons Fractals. 2020;140.

16. Hassanien AE, Salama A, Darwish A. Artificial Intelligence Approach to Predict the COVID-19 Patient's Recovery. No 3223 EasyChair. 2020.

17. Jin C, Chen W, Cao Y, Xu Z, Zhang X, Deng L, et al. Development and Evaluation of an AI System for COVID-19 Diagnosis. medRxiv. 2020 .

18. Almeshal AM, Almazrouee AI, Alenizi MR, Alhajeri SN. Forecasting the Spread of COVID-19 in Kuwait Using Compartmental and Logistic Regression Models. Appl Sci. 2020;10(10):3402.

19. Ali SM, Giordano R, Lakhani S, Walker DM. A review of randomized controlled trials of medical record powered clinical 
decision support system to improve the quality of diabetes care. Int $\mathrm{J}$ Med Inform. 2016;87:91-100.

20. Chin DL, Wilson MH, Trask AS, Johnson VT, Neaves BI, Gojova A, et al. Repurposing Clinical Decision Support System Data to Measure Dosing Errors and Clinician-Level Quality of Care. J Med Syst. 2020;44(10).

21. Goodman-Meza D, Rudas A, Chiang JN, Adamson PC, Ebinger J, Sun $\mathrm{N}$, et al. A machine-learning algorithm to increase COVID-19 inpatient diagnostic capacity. PLoS One. 2020;15(9):e0239474.

22. Parchure P, Joshi H, Dharmarajan K, Freeman R, Reich DL, Mazumdar $\mathrm{M}$, et al. Development and validation of a machine learning-based prediction model for near-term in-hospital mortality among patients with COVID-19. BMJ Support Palliat Care. 2020.

23. Alom MZ, Rahman M, Nasrin MS, Taha TM, Asari VK. COVID_MTNet: COVID-19 Detection with Multi-Task Deep Learning Approaches. arXiv preprint arXiv:200403747. 2020.

24. Hussain A, Bhowmik B, do Vale Moreira NC. COVID-19 and diabetes: Knowledge in progress. Diabetes Res Clin Pract. 2020;162.

25. Moujaess E, Kourie HR, Ghosn M. Cancer patients and research during COVID-19 pandemic: A systematic review of current evidence. Crit Rev Oncol Hematol. 2020;150:102972.

26. Chretien J-P, George D, Shaman J, Chitale RA, McKenzie FE. Influenza Forecasting in Human Populations: A Scoping Review. PLOS One. 2014;9(4):e94130.

27. Xu W, Zhao Y, Nian S, Feng L, Bai X, Luo X, et al. Differential analysis of disease risk assessment using binary logistic regression with different analysis strategies. J Int Med Res. 2018;46(9):3656-64.

28. Udhaya Kumar S, Thirumal Kumar D, Prabhu Christopher B, George Priya Doss C. The rise and impact of COVID-19 in India. Front Med. 2020;7.

29. Lalmuanawma S, Hussain J, Chhakchhuak L. Applications of Machine Learning and Artificial Intelligence for Covid-19 (SARSCoV-2) pandemic: A review. Chaos Solitons Fractals. 2020:110059.

30. Singer G, Marudi M. Ordinal Decision-Tree-Based Ensemble Approaches: The Case of Controlling the Daily Local Growth Rate of the COVID-19 Epidemic. Entropy. 2020;22(8):871.

31. Yoo SH, Geng H, Chiu TL, Yu SK, Cho DC, Heo J, et al. Deep learning-based decision-tree classifier for COVID-19 diagnosis from chest X-ray imaging. Front Med. 2020;7:427.

32. Lu W, Fu DL, Kong XX, Huang ZH, Hwang M, Zhu YS, et al. FOLFOX treatment response prediction in metastatic or recurrent colorectal cancer patients via machine learning algorithms. Cancer Medicine. Cancer Med. 2020;9(4):1419-1429.

33. Vinod DN, Prabaharan SRS. Data science and the role of Artificial Intelligence in achieving the fast diagnosis of Covid-19. Chaos Solitons Fractals. 2020;140:110182.

34. Elshazli RM, Torah EA, Elgaml A, El-Mowafy M, El-Misery M, Amin $\mathrm{MN}$, et al. Diagnostic and prognostic value of hematological and immunological markers in COVID-19 infection: A meta-analysis of 6320 patients. PloS One. 2020;15(8):e0238160.

35. Yeşilkanat CM. Spatio-temporal estimation of the daily cases of COVID-19 worldwide using a random forest machine-learning algorithm. Chaos Solitons Fractals. 2020;140:110210. 